\title{
Magnetic Noise Due to Sound of Footsteps on Wooden Free-Access Floor Outside a Magnetically Shielded Room for Biomagnetic and Nondestructive Measurements
}

\author{
K. Yamazaki ${ }^{1}$, T. Abe ${ }^{1}$, Y. Terazono ${ }^{2}$, N. Fujimaki ${ }^{2}$, T. Murata ${ }^{2}$, D. Oyama ${ }^{3}$, and K. Kobayashi ${ }^{3}$ \\ ${ }^{1}$ Takenaka Research and Development Institute, Takenaka Corporation, Chiba 270-1395, Japan \\ ${ }^{2}$ National Institute of Information and Communications Technology, Hyougo 651-2492, Japan \\ ${ }^{3}$ Faculty of Engineering, Iwate University, Iwate 020-8551, Japan
}

\begin{abstract}
In order to realize the ultimate low magnetic field in magnetically shielded rooms (MSRs) for nondestructive measurements and biomagnetic measurements such as magnetoencephalography and magnetocardiography, we investigated magnetic noises due to microtremors caused by sound pressure and mechanical vibration which arose from footsteps applied on the wooden free-access floor. Using superconducting quantum interference device (SQUID) magnetometers in this MSR, we measured the magnetic noises with and without operating an active microtremor isolation (ACMI) system that reduced microtremors transmitted from the concrete slab underneath the MSR. The microtremors of the MSR due to footsteps were divided into two components. One was caused by mechanical vibrations transmitted through the concrete slab at frequencies from 10 to $25 \mathrm{~Hz}$. Its frequency characteristic depended on the mechanical resonance of the MSR. The other was caused by sound pressure transmitted directly from the free-access floor. This component occurred at frequencies from 25 to $50 \mathrm{~Hz}$ even with the operation of ACMI when footsteps were applied on the wooden free-access floor.
\end{abstract}

Index Terms-Active microtremor isolation (ACMI) system, footstep, free-access floor, magnetically shielded room (MSR).

\section{INTRODUCTION}

B IOMAGNETIC measurements such as magnetoencephalography (MEG) and magnetocardiography (MCG) are usually carried out inside magnetically shielded rooms (MSRs) [1], [2]. The sensitivity of the measurements is limited by the residual magnetic noises in the MSR, which are caused by mechanical microtremors as well as by environmental magnetic noises, such as those generated by electric trains [3], motorcars, and electrical devices at low frequencies. To reduce magnetic noises due to microtremors caused by mechanical vibrations at frequencies less than $30 \mathrm{~Hz}$ [4], an active microtremor isolation (ACMI) system was installed in the basement underneath the MSR of the National Institute of Information and Communications Technology (NiCT).

In hospitals and research institute facilities, MSRs for biomagnetic and nondestructive measurements are often placed on concrete floors and surrounded by free-access floors made of wood and aluminum to conceal the cables underneath them and align the floor levels outside and inside the MSR. In the $\mathrm{NiCT}$, although microtremors transmitted from the concrete floor could be reduced by the ACMI [4], magnetic noises at frequencies higher than $10 \mathrm{~Hz}$ were still detected by SQUID magnetometers in the MSR when footsteps were applied on the wooden free-access floor. These magnetic noises were assumed to be produced by microtremors of the wall of the MSR that could be caused by sound pressure.

In order to realize the ultimate low magnetic field in the MSR, this paper reports an experimental investigation of the magnetic noise due to the sound pressure and mechanical vibrations

Manuscript received March 06, 2009. Current version published September 18, 2009. Corresponding author: K. Yamazaki (e-mail: yamazaki.takayuki@takenaka.co.jp).

Color versions of one or more of the figures in this paper are available online at http://ieeexplore.ieee.org.

Digital Object Identifier 10.1109/TMAG.2009.2025185 caused by footsteps. We measured the noise using SQUID magnetometers in the MSR. Clarifying the characteristics of such magnetic noises will help us in reducing them.

In this paper, we compared frequency spectra of the magnetic noise and vibration of an MSR with and without the operation of ACMI. First, magnetic noises and vibrations were measured without footsteps. Second, they were measured by applying controlled footsteps on the free-access floor, which included sound pressure and mechanical vibrations. Third, they were measured when only the mechanical vibration was applied to the MSR by controlled footsteps on the concrete slab under the free-access floor. Last, the relation between the sound pressure of footsteps on the free-access floor and magnetic noise was investigated quantitatively.

\section{Methods of Measurement}

We used an MEG system with its MSR supported by the ACMI (Fig. 1). Magnetic noise ( $B_{x}, B_{y}$, and $B_{z}$ ) was measured using the three SQUID magnetometers of the MEG system. Vibration was measured for the walls inside the MSR $\left(L_{w x}\right.$ and $\left.L_{w y}\right)$, and the ceilings outside the MSR $\left(A_{r x}\right.$ and $\left.A_{r y}\right)$. An ordinary acceleration sensor was used outside the MSR, while a laser vibrometer (PPV100) was used inside the MSR to avoid the magnetic noise. Sound pressure was measured inside and outside the MSR ( $S_{\text {in }}$ and $S_{\text {out }}$ ). The MSR consists of two permalloy layers (outer layer: $2 \mathrm{~mm}$ thick, inner layer: $3 \mathrm{~mm}$ ) and one aluminum layer $(9 \mathrm{~mm})$. A wide flange shape $(\mathrm{H}$-shaped $150 \mathrm{~mm} \times 150 \mathrm{~mm}$ ) made of aluminum was used as columns and beams (frame). Its shielding factor was about $40 \mathrm{~dB}$ at a frequency of $0.1 \mathrm{~Hz}$.

The frequency spectra of the vibration, magnetic noise, and sound pressure with and without the ACMI (ACMI-ON and -OFF) and with and without footsteps (footsteps-ON and -OFF) were compared to distinguish the components of magnetic noises due to sound pressure caused by footsteps [Fig. 1(b)] 


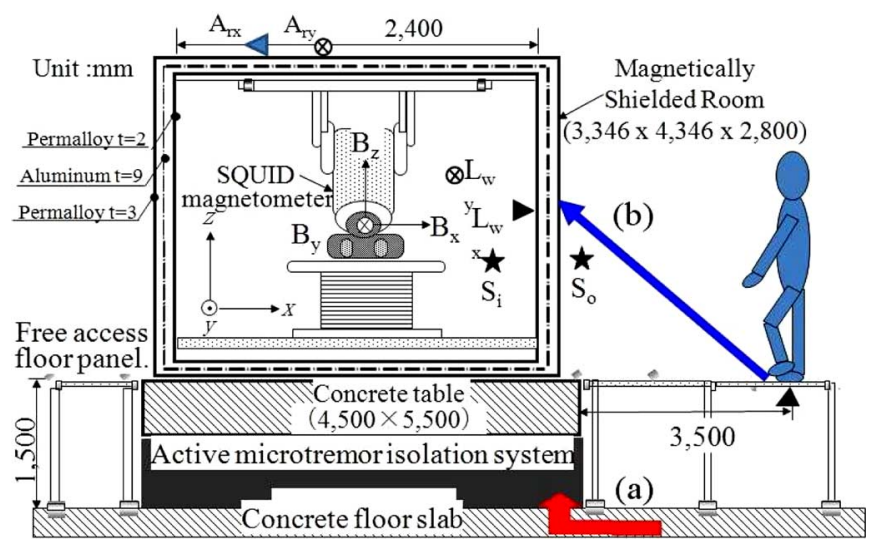

Fig. 1. MSR supported by an active microtremor isolation system and freeaccess floors, and points for measuring vibration and sound pressure.

from those caused by mechanical vibrations [Fig. 1(a)]. Sampling frequency was $1280 \mathrm{~Hz}$ and averaging time was eight.

\section{RESULTS AND DISCUSSION}

\section{A. Magnetic Noises due to Microtremors Caused by Environmental Vibrations}

Fig. 2 shows the measured frequency spectra of the velocity of the wall inside the MSR $L_{w x}$ and the magnetic noise $B_{y}$ without footsteps. Peaks of magnetic noise $B_{y}$ and $L_{w x}$ at frequencies from 7 to $25 \mathrm{~Hz}$ were smaller with ACMI-ON than with ACMIOFF. With ACMI-OFF, the coherence coefficients (CCs) were very high between $L_{w x}$ and $B_{y}$ at frequencies of about 13.6 and $18.9 \mathrm{~Hz}$, and between $L_{w y}$ and $B_{x}$ (figures were omitted) at frequencies of about 13.6 and $16.8 \mathrm{~Hz}$. With ACMI-OFF, the measured acceleration of the ceiling outside the MSR $A_{r x}$ and $A_{r y}$ had peaks of 16.8 and $18.9 \mathrm{~Hz}$ (figures were omitted). Those were assumed to reflect the mechanical resonance properties (MRPs) of the frame of the MSR, as predicted by modal analysis [5]. Furthermore, the other peak at $13.6 \mathrm{~Hz}$ was considered to be due to the MRP of the panels of the MSR. This suggests that the ACMI could primarily reduce magnetic noises due to microtremors caused by environmental vibrations depending on the MRPs of the frame and panels of the MSR at frequencies from 10 to $25 \mathrm{~Hz}$.

\section{B. Magnetic Noises due to Microtremors Caused by Footsteps on the Wooden Free-Access Floor Outside an MSR}

Fig. 3 shows the measured frequency spectra of the magnetic noise $B_{x}$, when controlled footsteps were applied on the free-access floor (with footsteps-ON) and were not applied (with footsteps-OFF) with ACMI-ON. Although microtremors transmitted from the floor should have been reduced by the ACMI, magnetic noises due to the footsteps remained at frequencies from 10 to $90 \mathrm{~Hz}$.

Fig. 4 shows the measured frequency spectra of the magnetic noise $B_{x}$, the velocity of the wall inside the MSR $L_{w x}$, and the sound pressure outside the MSR $\left(S_{\text {out }}\right)$ with footsteps-ON, for ACMI-ON and -OFF, and with footsteps-OFF for ACMI-OFF.

A peak of the magnetic noise $B_{x}$ at frequencies from 10 to $25 \mathrm{~Hz}$, was larger for ACMI-OFF and footsteps-ON, than for

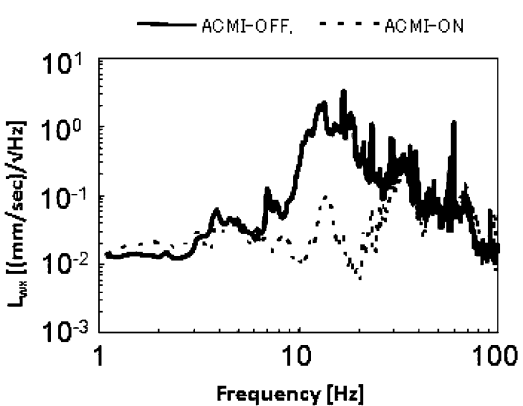

(a)

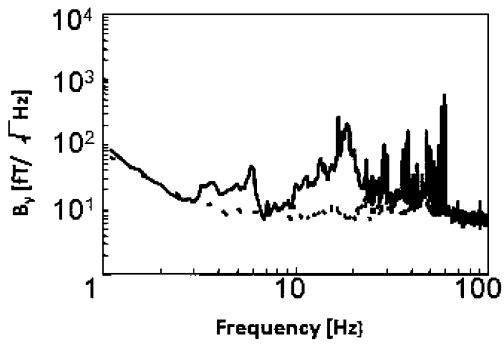

(b)

Fig. 2. Measured frequency spectra of velocity of the wall inside the MSR $L_{w x}$ and magnetic noise $B_{y}$.

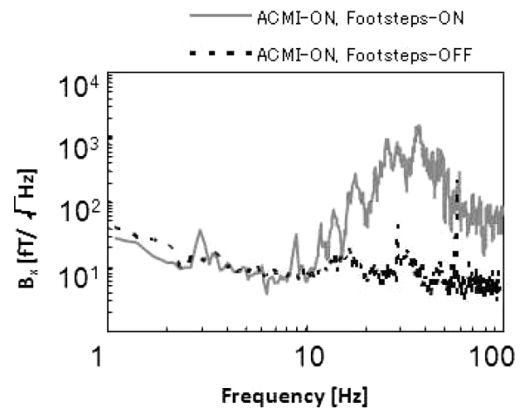

Fig. 3. Measured frequency spectra of magnetic noise $B_{x}$ when controlled footsteps were applied on the free-access floor with footsteps-ON and footstepsOFF with ACMI-ON.

ACMI-OFF and footsteps-OFF, and for ACMI-ON and footstepsON, showing that the magnetic noise caused by mechanical vibration transmitted from the concrete floor [Fig. 1(a)] was larger than that caused by sound pressure [Fig. 1(b)]. With footsteps ON, the $L_{w x}$ and $B_{x}$ with ACMI-ON and those with ACMI-OFF at frequencies higher than about $25 \mathrm{~Hz}$ were almost the same, suggesting that the magnetic noise caused by sound pressure was greater than that caused by mechanical vibration.

$S_{\text {out }}$ and $S_{\text {in }}$ (figures are omitted) due to footsteps had peaks at frequencies from 10 to $90 \mathrm{~Hz}$.

With ACMI-OFF, the CCs were very high between $S_{\text {out }}$ and $B_{x}$ at frequencies from about 20 to $40 \mathrm{~Hz}$, and between $S_{\text {out }}$ and $L_{w x}$ at frequencies from about 25 to $40 \mathrm{~Hz}$. But, the CCs were also very high between $B_{x}$ and $L_{w x}$ at frequencies of about 13.6 and $18.9 \mathrm{~Hz}$, which was considered to reflect the MRPs of the frame and panels of the MSR. With ACMI-ON, the CCs were very high between $S_{\text {out }}$ and $B_{x}$, and between $S_{\text {out }}$ and $L_{w x}$ at frequencies from about 15 to $40 \mathrm{~Hz}$. The frequency bandwidth of high CC with ACMI-OFF was narrower than that with ACMI-ON. This result shows that the magnetic noise and vibration of the wall with ACMI-OFF were accompanied by mechanical vibration but not the sound pressure. 


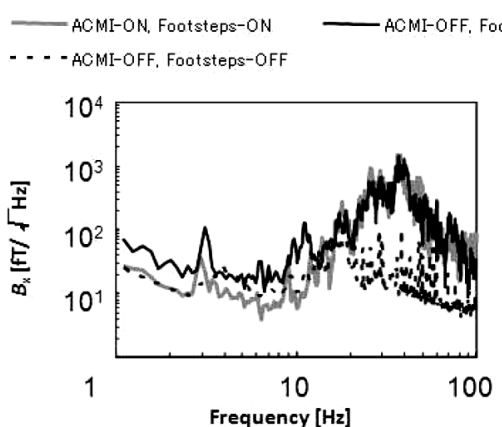

(a).

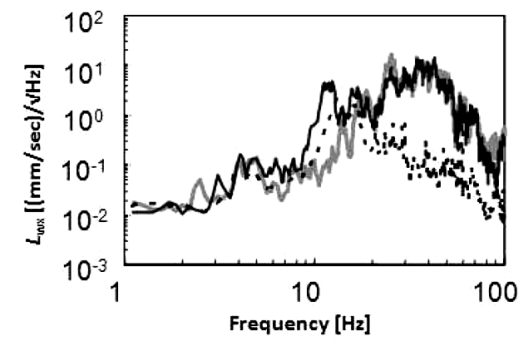

(b)

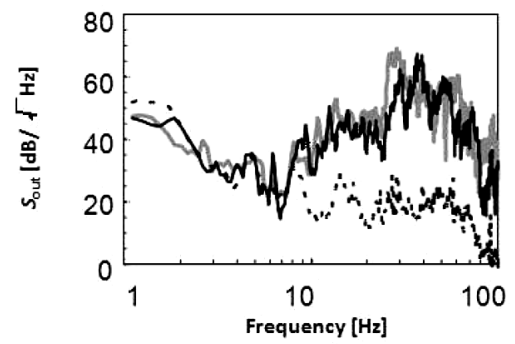

(c)

Fig. 4. Measured frequency spectra of magnetic noise $B_{x}$ and $B_{y}$, velocity of the wall inside the MSR $L_{w x}$, and sound pressure outside the MSR $S_{\text {out }}$, with footsteps-ON with ACMI-ON and -OFF, and with footsteps-OFF with ACMI-OFF. (a) $B_{x}$. (b) $L_{w x}$. (c) $S_{\text {out }}$.

\section{Magnetic Noises due to Microtremors Caused by Footsteps on Concrete Slab Outside an MSR}

Fig. 5 shows the measured frequency spectra of the magnetic noise $B_{x}$ and the velocity of the wall inside the MSR $L_{w x}$, when controlled footsteps were applied on the concrete slab (with footsteps-ON, concrete slab) with ACMI-ON and ACMI-OFF and on the free-access floor (footsteps-ON, free-access floor) with ACMI-OFF. With ACMI-OFF, the $B_{x}$ and $L_{w x}$ differed between footsteps-ON, free-access floor and footsteps-ON, concrete slab at frequencies higher than about $25 \mathrm{~Hz}$. This indicates that the vibration of the walls and magnetic noise due to the sound pressure on the free-access floor are more significant than that due to mechanical vibration through the concrete floor. Peaks of $L_{w x}$ at frequencies below $25 \mathrm{~Hz}$ were higher than those above $25 \mathrm{~Hz}$ (with footsteps-ON, concrete slab), showing that the resonance frequencies of the MSR were below $25 \mathrm{~Hz}$. At frequencies higher than about $10 \mathrm{~Hz}, B_{x}$ and $L_{w x}$ were smaller with ACMI-ON than with ACMI-OFF. These results show that magnetic noises from mechanical vibrations due to footsteps on the concrete slab were mostly reduced by the ACMI.

\section{Discussion}

The present results suggest that the magnetic noise due to footsteps on the wooden free-access floor with ACMI-OFF de-

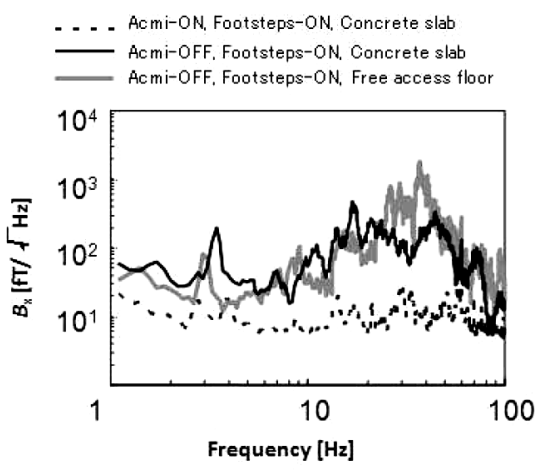

(a)

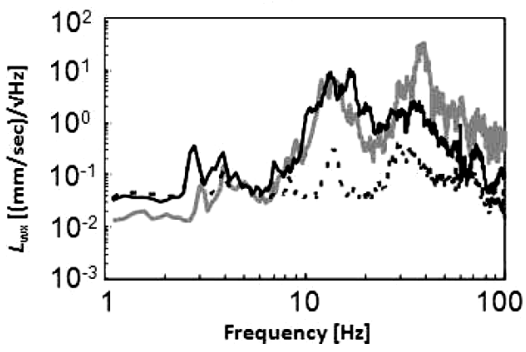

(b)

Fig. 5. Measured frequency spectra of magnetic noise $B_{x}$ and velocity of the wall inside the MSR $L_{w x}$, when controlled footsteps were applied on the concrete slab (with footsteps-ON, concrete slab) with ACMI-ON and ACMI-OFF and on the free-access floor (with footsteps-ON, free-access floor) with ACMI-OFF. (a) $B_{x}$. (b) $L_{w x}$.

pends on mechanical vibrations at frequencies from about 10 to $25 \mathrm{~Hz}$, and on sound pressure at frequencies between 25 and $50 \mathrm{~Hz}$. Thus, these frequency bandwidths separated the components of magnetic noises due to the sound pressure from footsteps [Fig. 1(b)] from those caused by mechanical vibrations [Fig. 1(a)]. This can be illustrated as follows. The sound pressure due to footsteps on the free-access floor was larger at higher than $25 \mathrm{~Hz}$. As shown in Fig. 2, the MRPs of the MSR appeared at frequencies from 10 to $25 \mathrm{~Hz}$. Thus, the MSR was susceptible to mechanical vibration at these frequencies, but was not susceptible at frequencies higher than $25 \mathrm{~Hz}$. As a result, the ACMI could effectively eliminate magnetic noise and vibration of the wall between 10 and $25 \mathrm{~Hz}$, as shown in Fig. 5. However, ACMI could not reduce the magnetic noises due to footsteps at frequencies from 25 to $50 \mathrm{~Hz}$. They are considered to be caused via sound pressure. This suggests that it is important to suppress occurrence of sound noises or attenuate them during biomagnetic measurements in the MSR even when using ACMI.

\section{Relation Between Sound PRessure AND MAGNETIC NOISE}

At frequencies from 15 to $40 \mathrm{~Hz}$ with ACMI-ON, the CCs between the sound pressure outside the MSR $S_{\text {out }}$ and the velocity of the walls inside the MSR, $L_{w x}$ and those between the magnetic noise $B_{x}$ and $S_{\text {out }}$ were high. We investigated the relation between these quantities.

Fig. 6 shows the relation between $S_{\text {out }}[\mathrm{Pa}]$ computed from $S_{\text {out }}[\mathrm{dB}]$ and the displacement $\Delta L_{w x}$ of the inner wall in the perpendicular direction obtained from the measured velocity $L_{w y}$ for 23 frequencies from 15 to $40 \mathrm{~Hz}$ (the frequencies where the CC was lower than 0.9 were excluded). $\Delta L_{w x}$ is approxi- 


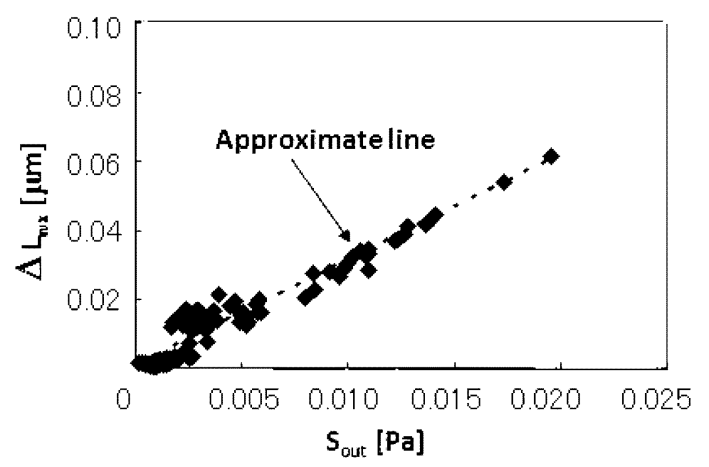

Fig. 6. Relationship between $S_{\text {out }}[\mathrm{Pa}]$ and the displacement of the inner wall in perpendicular direction $\Delta L_{w x}$.

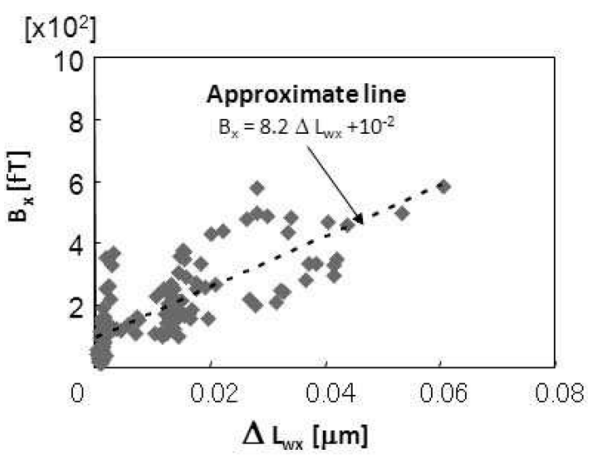

Fig. 7. Relationship between the displacement of the inner wall in perpendicular direction $\Delta L_{w x}$ obtained from $L_{w x}$ and magnetic noise $B_{x}$.

mately proportional to the sound pressure $S_{\text {out }}$. This depends on the construction of the MSR including frames, columns, beams, and shielding panels.

Fig. 7 shows the relation between the displacement $\Delta L_{w x}$ of the inner wall in the perpendicular direction obtained from $L_{w y}$ and the magnetic noises $B_{x}$ for 23 frequencies from 15 to $40 \mathrm{~Hz}$ (the frequencies where the $\mathrm{CC}$ was lower than 0.9 were excluded). It was demonstrated that $B_{x}$ is approximately proportional to $\Delta L_{w x}$. In this MSR, a $1-\mu \mathrm{m}$ displacement of the inner wall in the perpendicular direction resulted in about $8 \mathrm{pT}$ of magnetic noise.

Fig. 8 shows the relation between $S_{\text {out }}[\mathrm{Pa}]$ and $B_{x}$ for 56 frequencies from 15 to $40 \mathrm{~Hz}$ (the frequencies where the $\mathrm{CC}$ was lower than 0.9 were excluded). This shows the tendency for magnetic noise $B_{x}$ to increase as the sound pressure outside due to footsteps on the wooden free-access floor increases. Magnetic noise of 200 and $600 \mathrm{fT}$ corresponded to $0.005 \mathrm{~Pa}(50 \mathrm{~dB})$ and $0.2 \mathrm{~Pa}(60 \mathrm{~dB})$, respectively.

\section{CONCLUSION}

An experimental investigation of the magnetic noise due to the sound pressure and mechanical vibrations caused by footsteps on the free-access floor was carried out by comparing the conditions where an ACMI in the basement underneath the MSR was operated or not operated.

1) Without footsteps, the ACMI could mainly reduce magnetic noises due to microtremors caused by mechanical vibrations depending on the mechanical resonance properties

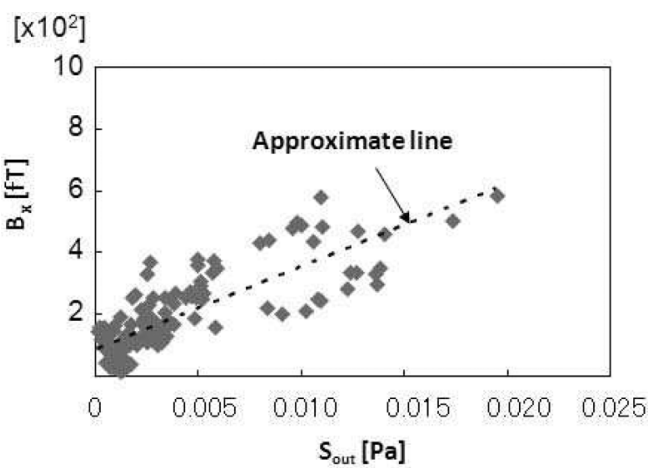

Fig. 8. Relationship between $S_{\text {out }}[\mathrm{Pa}]$ and magnetic noise $B_{x}$.

of the frame and panels of the MSR at frequencies from 10 to $25 \mathrm{~Hz}$.

2) When footsteps were applied on the wooden free-access floor, the ACMI effectively eliminated magnetic noise due to microtremors caused by mechanical vibrations between 10 and $25 \mathrm{~Hz}$, but not those caused by sound pressure between 25 and $50 \mathrm{~Hz}$. The microtremors transmitted from the concrete floor depended on the mechanical resonance properties of the frame and panels of the MSR, but those directly transmitted from free-access floor did not.

3) The displacement of the wall was approximately proportional to the sound pressure caused by footsteps and the magnetic noise was approximately proportional to the displacement of the wall. Thus, the magnetic noise was approximately proportional to the sound pressure. That is, 1 $\mu \mathrm{m}$ of wall displacement resulted in about $8 \mathrm{pT}$ of magnetic noise. Magnetic noise of 200 and $600 \mathrm{fT}$ corresponded to about $0.005 \mathrm{~Pa}(50 \mathrm{~dB})$ and $0.2 \mathrm{~Pa}(60 \mathrm{~dB})$, respectively.

4) The implications of the present results are summarized as follows. Both mechanical vibration and sound pressure produce magnetic noises in the MSR, which may deteriorate the biomagnetic measurements such as MEG. The noises due to them have different frequency components, depending on the mechanical properties of the MSR and the constructions related to sound pressures. It is effective to suppress these mechanical vibrations and sound noises for minimizing the magnetic noises during biomagnetic measurements.

\section{REFERENCES}

[1] J. Vrba, J. McCubbin, and S. E. Robinson, "Vibration analysis of MEG systems," in Proc. 11th Int. Conf. Biomagn., 1998, pp. 1239-1242.

[2] M. Burghoff, T. H. Sander, A. Schnabel, D. Drung, and L. Trahms, "dc magnetoence phalography: Direct measurement in a magnetically shielded room," Appl. Phys. Lett., vol. 85, no. 25, pp. 6278-6280, Dec. 2004.

[3] K. Yamazaki, K. Kato, K. Kobayashi, Y. Uchikawa, Y. Kumagai, A. Haga, and K. Fujiwara, "Characteristics and prediction of magnetic noise due to DC electric railcars for biomagnetic measurement," IEEE Trans. Magn., vol. 37, no. 4, pp. 2884-2887, Jul. 2001.

[4] T. Abe, K. Yamazaki, S. Miayauchi, N. Fujimaki, and K. Kobayashi, "Reduction of magnetic noise of magnetically shielded room by active microtremor isolation systems," (in Japanese) IEEJ Trans. Fundam. Mater., vol. 119-A, no. 12, pp. 1474-1480, 1999.

[5] C. C. Kennedy and C. D. P. Pancu, "Use of vectors in vibration measurement and analysis," J. Aeronaut. Sci., vol. 14, no. 11, p. 603, 1947. 